\title{
On network representations of antennas inside resonating environments
}

\author{
F. Gronwald, S. Glüge, and J. Nitsch
}

Institute for Fundamental Electrical Engineering and EMC, Otto-von-Guericke-University Magdeburg, Universitätsplatz 2, 39106 Magdeburg, Germany

\begin{abstract}
We discuss network representations of dipole antennas within electromagnetic cavities. It is pointed out that for a given configuration these representations are not unique. For an efficient evaluation a network representation should be chosen such that it involves as few network elements as possible. The field theoretical analogue of this circumstance is the possibility to express electromagnetic cavities' Green's functions by representations which exhibit different convergence properties. An explicit example of a dipole antenna within a rectangular cavity clarifies the corresponding interrelation between network theory and electromagnetic field theory. As an application, current spectra are calculated for the case that the antenna is nonlinearly loaded and subject to a two-tone excitation.
\end{abstract}

\section{Introduction}

In Electromagnetic Compatibility (EMC) analysis it is often useful to turn an electromagnetic field problem into an equivalent network problem (Lee, 1995). This step usually requires to solve the electromagnetic field problem in order to explicitly calculate equivalent network parameters. Ideally, the solution of a modeled problem consists of calculated network parameters that are accessible to direct measurements, such as scattering parameters that can be measured by means of a network analyzer.

The advanced concept of Electromagnetic (EM) Topology exemplifies the conversion of an electromagnetic field problem into a network problem. In EM topology complex systems are divided into smaller subsystems and dominant coupling paths are identified, as schematically drawn in Fig. 1. These coupling paths are described by a Baum-Liu-Tesche (BLT) network with BLT equations as corresponding dynam- ical network equations (Tesche et al., 1997). In this framework subsystems reduce to transducers, power transfer elements, or coupling elements and are characterized by appropriate network representations.

We will concentrate in the following on network representations of dipole antennas inside resonating environments, such as dipole antennas that are located within the interior of an electromagnetic cavity. Quite generally, dipole antennas can serve as models for Electromagnetic Interference (EMI) sources or victims. Since EMI sources or victims often are enclosed by metallic boundaries, which can resemble electromagnetic cavities, one would like to be able to characterize antenna properties not only in free space ("exterior problem") but also in resonating environments ("interior problem").

Typically, network representations of antennas are given by impedance functions which are defined via voltages and currents at the antenna inputs (Elliott, 1981). The concept of impedance is applicable to both single antennas or systems of antennas. As an example, Fig. 2 displays two coupled antennas which are characterized by an impedance matrix.

In what follows we will mainly be concerned with the characterization and calculation of antenna impedances that relate to antennas within electromagnetic cavities. Recently, in an EMC context this topic has been investigated in a systematic way (Gronwald, 2006). In the context of antenna theory it is not common to place antennas within cavities, simply because antennas are designed to radiate in free space. However, there are exceptions such as the Wheeler's cap technique which serves to measure antenna efficiencies within a cavity. In the application of this technique, network representations of antennas are of interest, too (Huang et al., 2005).

Correspondence to: F. Gronwald

(frank.gronwald@et.uni-magdeburg.de)

Published by Copernicus Publications on behalf of the URSI Landesausschuss in der Bundesrepublik Deutschland e.V. 


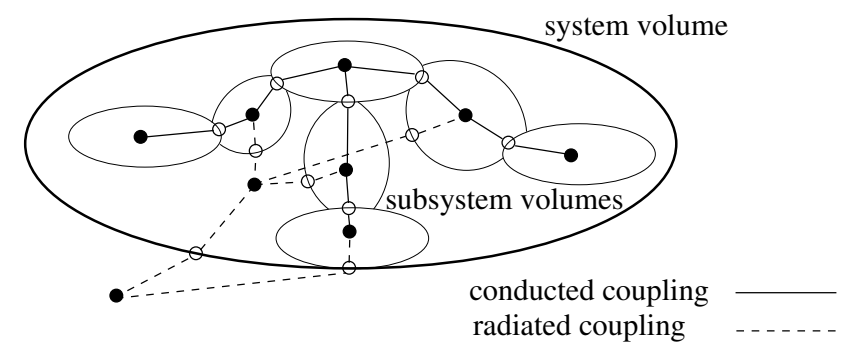

Fig. 1. General example of a BLT network which consists of various coupling paths. It is common to distinguish between conducted coupling and radiated coupling.

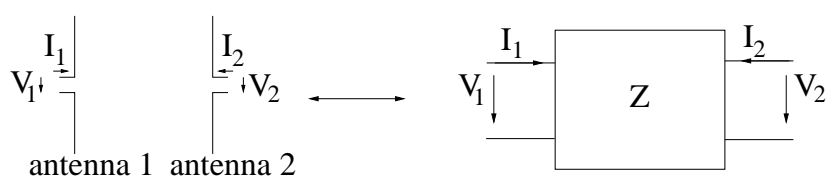

Fig. 2. Network representation of two coupled antennas by the impedance matrix of a two-port.

\section{Network representation of impedance functions}

General and useful information on impedance functions can be obtained from complex analysis. This requires to view impedance functions as functions in the complex frequency plane where the complex frequency is given by the Laplacetransform variable $s=\Omega+j \omega$.

\subsection{Systems with finite number of natural frequencies}

In an early paper (Foster, 1924), Foster has shown that the impedance of a lossless one-port with a finite number of inductances and capacitances is of the form

$$
\begin{aligned}
& Z(\omega)=-\frac{j}{\omega C_{0}}+j \omega L_{\infty}+\sum_{n=1}^{N} \frac{j \omega}{C_{n}\left(\omega_{n}^{2}-\omega^{2}\right)} \\
& =\frac{a_{0}\left(1-\omega / \omega_{z_{1}}\right)\left(1-\omega / \omega_{z_{2}}\right) \cdots}{b_{0}\left(1-\omega / \omega_{p_{1}}\right)\left(1-\omega / \omega_{p_{2}}\right) \cdots},
\end{aligned}
$$

compare Fig. 3, and it is seen that the impedance is largely determined by its zeros $\omega_{z_{n}}$ and poles $\omega_{p_{n}}$. Mathematically, this is a consequence of the Mittag-Leffler theorem.

Antennas within electromagnetic cavities constitute electromagnetic systems with an infinite number of natural frequencies. Therefore the result of Foster cannot be directly applied to our analysis.

\subsection{Systems with infinite number of natural frequencies}

Schelkunoff showed that Foster's network representation can be generalized to systems with an infinite number of natural frequencies (Schelkunoff, 1944). It turned out that the resulting network representation is not unique. We have, for

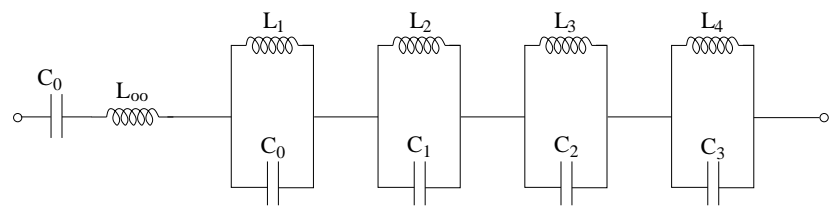

Fig. 3. Impedance of a lossless one-port which represents an electromagnetic system with a finite number of natural frequencies when the input terminals are open. The number of parallel circuits is equal to the number of natural frequencies.

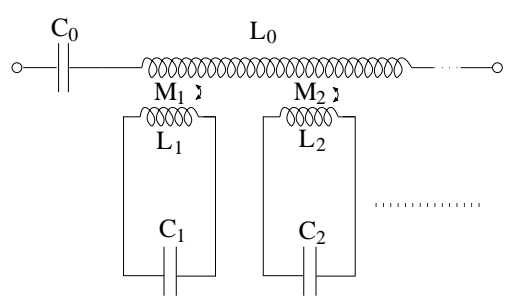

Fig. 4. Alternative network representation of an electromagnetic system with an infinite number of natural frequencies.

example,

$$
\begin{aligned}
& Z(\omega)=j \omega L_{\infty}-\frac{j}{\omega C_{0}}+\sum_{n=1}^{\infty} \frac{j \omega}{C_{n}\left(\omega_{n}^{2}-\omega^{2}\right)} \\
& =j \omega L_{0}-\frac{j}{\omega C_{0}}+\sum_{n=1}^{\infty} \frac{\omega_{n}^{2} M_{n}^{2}}{L_{n}}\left(\frac{j \omega}{\omega_{n}^{2}-\omega^{2}}-\frac{j \omega}{\omega_{n}^{2}}\right) .
\end{aligned}
$$

Clearly, Eq. (3) is a direct generalization of Eq. (1). The fact that Eq. (4) is equivalent to Eq. (3) is not immediate (Schelkunoff, 1944). We display in Fig. 4 the network representation that corresponds to the mathematical expression in Eq. (4). The series in Eq. (4) often is prefered over the series in Eq. (3) because it exhibits better convergence properties. As a result, less network elements are required to model a specific impedance.

\subsection{Microwave networks}

The above concepts are directly applicable to microwave networks and other resonating electromagnetic structures such as cavities, waveguides or transmission lines. Besides the general information that is provided by complex analysis it is then also required to apply Maxwell's theory and investigate the modal properties of the electromagnetic field within the resonating environment. Explicit values for the circuit elements that appear in network representations, such as Eq. (3) or Eq. (4), are obtained by the solution of Maxwell's equations. In this context, a standard method is to take advantage of the Poynting theorem and calculate the wanted network parameters via the electromagnetic energy that is stored in each mode (Ramo et al., 1994). 
This and related methods yield network representations of the resonating environment itself, like network representations of an empty cavity. They do not include the interaction of the electromagnetic field with additional sources or scatterers, like the interaction with an antenna.

\subsection{Antennas}

Similar to Eq. (2) and its generalization to an infinite number of natural frequencies the input impedance of an antenna in free space can be described by a pole/zero expansion (Baum, 2002). For a dipole antenna in free space one has an expansion of the form

$$
\begin{aligned}
& Z(\omega)=\frac{\left(1-\omega / \omega_{z_{1}}\right)\left(1+\omega / \omega_{z_{1}}\right)\left(1-\omega / \omega_{z_{2}}\right) \cdots}{j \omega C\left(1-\omega / \omega_{p_{1}}\right)\left(1+\omega / \omega_{p_{1}}\right)\left(1-\omega / \omega_{p_{2}}\right) \cdots} \\
& \approx \frac{1}{j \omega C}+R+j \omega L-A \omega^{2}+\cdots
\end{aligned}
$$

Unfortunately, this mathematical expression has no exact representation by a common $R L C$ network. Equivalent circuit models follow from the Singularity Expansion Method and involve complex circuit elements (Baum, 1976). The interpretation and use of these complex circuit elements is not immediate. We have a similar situation in the study of generalized transmission line models which exhibit complex transmission line parameters if radiation phenomena need to be taken into account (Haase et al., 2003, 2004).

\subsection{Antennas inside electromagnetic cavities}

So far we have discussed two points:

- Resonating microwave structures, such as cavities or waveguides, have exact network representations of the form Eq. (3) or Eq. (4) ${ }^{1}$. These representations do not take into account the interaction of the electromagnetic field with additional sources or scatterers.

- Antennas do not have exact network representations in terms of $R L C$ network elements. This is due to the fact that these elements cannot properly take into account radiation effects. However, low-frequency expansions of the form Eq. (6) are meaningful and take into account radiation effects in an approximate way.

We will now focus on antennas inside electromagnetic cavities and merge cavity properties with antenna properties. To this end, we note that, quite generally, antenna impedances are of the form (Elliott, 1981)

$Z_{\text {self }}=-\frac{\langle\boldsymbol{E}, \boldsymbol{J}\rangle_{p}}{I^{2}}$,

$Z_{12}=-\frac{\left\langle\boldsymbol{E}^{b}, \boldsymbol{J}^{a}\right\rangle_{p}}{I_{2}^{a} I_{1}^{b}}$

\footnotetext{
${ }^{1}$ The representations are exact as long as Ohmic losses are neglected. Small losses can be acounted for in a perturbative way by the inclusion of Ohmic resistors.
}

with $Z_{\text {self }}$ a self impedance and $Z_{12}$ a mutual impedance between two antennas. The currents $I, I_{2}^{a}$, and $I_{1}^{b}$ denote antenna input currents, $\boldsymbol{E}, \boldsymbol{E}^{b}$ denote electric fields, and $\boldsymbol{J}, \boldsymbol{J}^{a}$ are electric current densities. We also introduced for notational convenience a pseudo inner product which, for vector functions $\boldsymbol{f}, \boldsymbol{g}$ that are integrable over a domain $\Gamma$, can be taken as

$$
\langle\boldsymbol{f}, \boldsymbol{g}\rangle_{p}:=\int_{\Gamma} \boldsymbol{f}(\boldsymbol{r}) \cdot \boldsymbol{g}(\boldsymbol{r}) d \Gamma .
$$

The formulas (7) and (8) are valid both in free space and within a cavity as long as reciprocity is satisfied. If an inner product is defined by

$$
\langle\boldsymbol{f}, \boldsymbol{g}\rangle:=\int_{\Gamma} \boldsymbol{f}(\boldsymbol{r}) \cdot \boldsymbol{g}^{*}(\boldsymbol{r}) d \Gamma,
$$

with the asterisk indicating the complex conjugate, the electric field $\boldsymbol{E}$ that is generated by a current density $\boldsymbol{J}$ can be written as

$\boldsymbol{E}(\boldsymbol{r})=\left\langle\overline{\boldsymbol{G}}^{E}, \boldsymbol{J}\right\rangle$,

with the dyadic electric Green's function $\overline{\boldsymbol{G}}^{E}$.

In a cavity, the dyadic electric Green's function can be expressed in terms of a modal representation with longitudinal eigenmodes $\boldsymbol{L}_{n}$ and transverse eigenmodes $\boldsymbol{F}_{n}$ (Collin, 1991) which fulfill

$$
\begin{aligned}
& \nabla \times \nabla \times \boldsymbol{L}_{n}=\mathbf{0}, \\
& \nabla \times \nabla \times \boldsymbol{F}_{n}=k_{n}^{2} \boldsymbol{F}_{n} .
\end{aligned}
$$

As a consequence, the electric field has the modal representation

$$
\boldsymbol{E}=\frac{j \omega \mu}{k^{2}} \sum_{n}\left\langle\boldsymbol{J}, \boldsymbol{L}_{n}\right\rangle \boldsymbol{L}_{n}+j \omega \mu \sum_{n} \frac{\left\langle\boldsymbol{J}, \boldsymbol{F}_{n}\right\rangle}{k^{2}-k_{n}^{2}} \boldsymbol{F}_{n} .
$$

with $\mu$ the absolute permeability and $k$ the wavenumber. If we insert this expansion into Eq. (8), for example, we find

$$
Z_{\text {self }}=-\frac{j \omega \mu}{k^{2} I^{2}} \sum_{n}\left\langle\boldsymbol{J}, \boldsymbol{L}_{n}\right\rangle^{2}-\frac{j \omega \mu}{I^{2}} \sum_{n} \frac{\left\langle\boldsymbol{J}, \boldsymbol{F}_{n}\right\rangle^{2}}{k^{2}-k_{n}^{2}} .
$$

It follows that single modes $\boldsymbol{F}_{n}$ are characterized by parallel $R L C$ circuits with

$$
\begin{aligned}
R_{n} & =\frac{Q\left\langle\boldsymbol{J}, \boldsymbol{F}_{n}\right\rangle^{2}}{\varepsilon I^{2} \omega_{n}}, \\
L_{n} & =\frac{\left\langle\boldsymbol{J}, \boldsymbol{F}_{n}\right\rangle^{2}}{\varepsilon\left(I \omega_{n}\right)^{2}}, \\
C_{n} & =\frac{\varepsilon I^{2}}{\left\langle\boldsymbol{J}, \boldsymbol{F}_{n}\right\rangle^{2}},
\end{aligned}
$$

where $\varepsilon$ denotes the absolute permittivity and $Q$ the quality factor of the cavity. This yields the desired relations between field quantities and network quantities. 


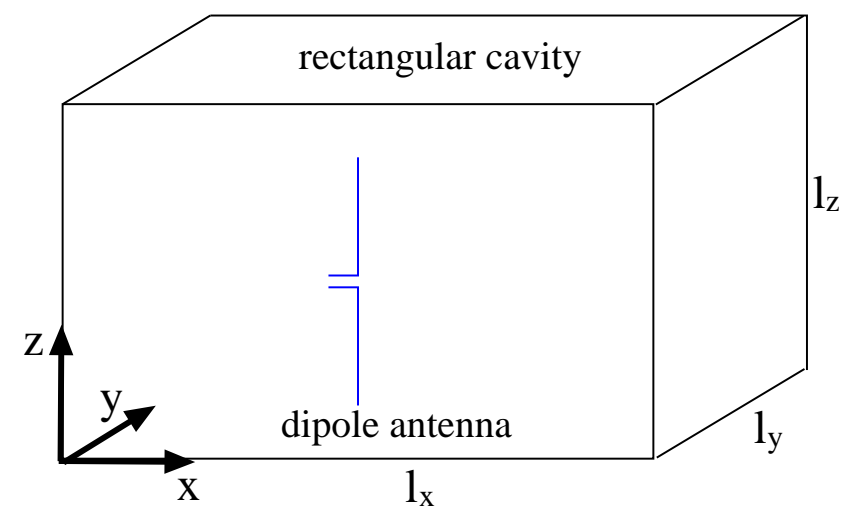

Fig. 5. Dipole antenna within a rectangular cavity.

\subsection{The problem of convergence}

Expansions of the form Eq. (14) or Eq. (15) have the disadvantage that usually they exhibit poor convergence properties. To improve this situation it is required to find representations of the electric Green's function which quickly converge both in the source region and at resonance. The fact that the representation of a cavities' Green's function is not unique constitutes the field-theoretical analogue to the possibility to choose various representations for the impedance function of a system with an infinite number of natural frequencies.

In the case of cavities' Green's functions it is often advantageous to split off the free space Green's function. We illustrate this by the example of a rectangular cavity: The corresponding dyadic Green's function $\overline{\boldsymbol{G}}^{A}$ for the magnetic vector potential $\boldsymbol{A}$ has a modal expansion of the form (Morse and Feshbach, 1953)

$\overline{\boldsymbol{G}}^{A}\left(\boldsymbol{r}, \boldsymbol{r}^{\prime}, k\right)=$

$\sum_{m, n, p=0}^{\infty} \frac{\epsilon_{0 m} \epsilon_{0 n} \epsilon_{0 p}}{l_{x} l_{y} l_{z}} \frac{1}{k_{m n p}^{2}-k^{2}}\left(f_{m n p}^{x}(\boldsymbol{r}) f_{m n p}^{x}\left(\boldsymbol{r}^{\prime}\right) \boldsymbol{e}_{x} \boldsymbol{e}_{x}+\right.$

$\left.f_{m n p}^{y}(\boldsymbol{r}) f_{m n p}^{y}\left(\boldsymbol{r}^{\prime}\right) \boldsymbol{e}_{y} \boldsymbol{e}_{y}+f_{m n p}^{z}(\boldsymbol{r}) f_{m n p}^{z}\left(\boldsymbol{r}^{\prime}\right) \boldsymbol{e}_{z} \boldsymbol{e}_{z}\right)$.

with $\epsilon_{0 N}$ the Neumann factor and $f_{m n p}^{x, y, z}$ the eigenfunctions that correspond to the Helmholtz equation for the magnetic vector potential $\boldsymbol{A}$. This expansion can be approximated by

$\overline{\boldsymbol{G}}^{A}\left(\boldsymbol{r}, \boldsymbol{r}^{\prime}, k\right) \approx \frac{1}{4 \pi} \frac{e^{-j k\left|\boldsymbol{r}-\boldsymbol{r}^{\prime}\right|}}{\left|\boldsymbol{r}-\boldsymbol{r}^{\prime}\right|} \overline{\boldsymbol{I}}+$

$\sum_{m, n, p}^{M, N, P} \frac{\epsilon_{0 m} \epsilon_{0 n} \epsilon_{0 p}}{l_{x} l_{y} l_{z}} \frac{1}{k_{m n p}^{2}-k^{2}}\left(f_{m n p}^{x}(\boldsymbol{r}) f_{m n p}^{x}\left(\boldsymbol{r}^{\prime}\right) \boldsymbol{e}_{x} \boldsymbol{e}_{x}+\right.$

$\left.f_{m n p}^{y}(\boldsymbol{r}) f_{m n p}^{y}\left(\boldsymbol{r}^{\prime}\right) \boldsymbol{e}_{y} \boldsymbol{e}_{y}+f_{m n p}^{z}(\boldsymbol{r}) f_{m n p}^{z}\left(\boldsymbol{r}^{\prime}\right) \boldsymbol{e}_{z} \boldsymbol{e}_{z}\right)$.

where the summation now only extends over those modes which, at the frequency range considered, are dominantly excited. In principle, all modes will contribute to the Green's

\begin{tabular}{ccc}
\hline free space & resonance 110 & resonance 130 \\
\hline$L_{\text {free }}=286.4 \mathrm{nH}$ & $L_{110}=12.65 \mathrm{nH}$ & $L_{130}=4.171 \mathrm{nH}$ \\
$C_{\text {free }}=2.652 \mathrm{pF}$ & $C_{110}=1.850 \mathrm{nF}$ & $C_{130}=1.278 \mathrm{nF}$ \\
- & $R_{110}=1500 \Omega$ & $R_{130}=1500 \Omega$ \\
\hline
\end{tabular}

\begin{tabular}{cc}
\hline resonance 310 & resonance 330 \\
\hline$L_{310}=3.469 \mathrm{nH}$ & $L_{330}=2.434 \mathrm{nH}$ \\
$C_{310}=1.202 \mathrm{nF}$ & $C_{330}=1.068 \mathrm{nF}$ \\
$R_{310}=1500 \Omega$ & $R_{330}=1500 \Omega$ \\
\hline
\end{tabular}

Table 1. Explicit values of the elements of the network representation in Fig. 6 as obtained from the method of moments.

function, but the majority of modes is required to model, by superposition, the Coulomb interaction which is contained in the Green's function of free space. Therefore, if we split off the Green's function of free space we split off the contribution of most of the relevant modes and express this contribution as a single term. This is, in short, the essential idea of using so-called hybrid representations of electromagnetic Green's function which separate the singular features of the Coulomb interaction from the summation of single modes (Felsen, 1984,G).

\section{Example and application}

We now illustrate the concepts outlined above by means of an example and an application to a nonlinearly loaded antenna.

\subsection{Dipole antenna within a rectangular cavity}

We consider a dipole antenna which is located within a rectangular cavity, compare Fig. 5. The cavity dimensions are $l_{x}=6 \mathrm{~m}, l_{y}=7 \mathrm{~m}, l_{z}=3 \mathrm{~m}$, the antenna is aligned to the $z$-axis and placed at $\boldsymbol{r}_{0}=\left(l_{x} / 2, l_{y} / 2, l_{z} / 2\right)$.

In the frequency range $25 \mathrm{MHz}$ to $100 \mathrm{MHz}$ the antenna will couple to the modes $110(32.9 \mathrm{MHz}), 130(68.9 \mathrm{MHz})$, $310(78.0 \mathrm{MHz})$, and $330(98.7 \mathrm{MHz})$. It is then, in this frequency range, sufficient to include in the expression (20) for the electromagnetic Green's function $\overline{\boldsymbol{G}}^{A}$ the eigenfunctions that correspond to these four modes. The error that is made by this approxiation can be shown to be very small. With this approximation a network representation of the dipole antenna is obtained which consist of only 14 elements and is shown in Fig. 6. The elements $C_{\text {free }}$ and $L_{\text {free }}$ take into account the near-field properties of the antenna that are mainly due to the Coulomb interaction. They correspond to the capacitance $C$ and inductance $L$ of the low-frequency expansion Eq. (6). The terms $R$ and $-A \omega^{2}$, respectively, are absent because the antenna is assumed to be lossless and be- 


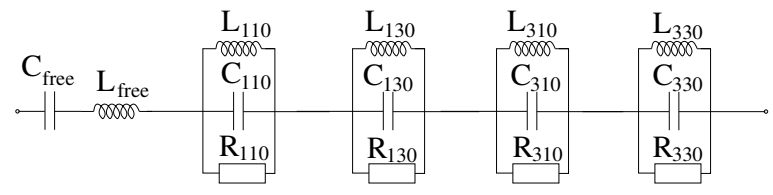

Fig. 6. Approximate network representation of the dipole antenna of Fig. 5 for the frequency range $25 \mathrm{MHz}$ to $100 \mathrm{MHz}$.

cause there are no radiation losses in our example. Explicit values for the network elements are given in Table 1. They follow from a method of moment solution which determines, for a given excitation at the antenna input, the resulting current distribution on the antenna. For the problem of calculating antenna impedances within cavities by the method of moment we refer to (Gronwald, 2005).

\subsection{Intermodulation effects of a nonlinear load}

The necessity to use network representations of electromagnetic systems becomes apparent if the effect of nonlinear elements needs to be taken into account. In this case it usually is not practicable to apply nonlinear field theory. Rather, the general strategy is to first transform the linear part of a nonlinear problem into a network representation. Then the nonlinear elements are included in the problem and the resulting problem is solved by means of nonlinear circuit theory.

For illustration we consider a dipole antenna within a rectangular cavity, as defined in the last subsection. We now assume that the antenna is loaded with a nonlinearity of the form

$v_{\mathrm{nl}}(t)=1 k \Omega i(t)-6 \frac{k \Omega}{\mathrm{A}} i^{2}(t)+3 \frac{k \Omega}{\mathrm{A}^{2}} i^{3}(t)$.

If we excite this antenna by a two-tone excitation we expect that intermodulation will occur. This phenomenon describes the generation of secondary frequencies from the electromagnetic excitation of nonlinearities by primary frequencies (Maas, 2003).

The network representation of Fig. 6 together with the explicit form Eq. (21) of the nonlinearity and a prescribed excitation defines a nonlinear circuit problem for the unknown antenna current. The corresponding network problem can be solved by standard methods. In this context we found the method of Picard iteration useful (Nitsch et al., 2005). This method, as is typical for a calculation method for nonlinear problems, is an iterative one, but it also is analytic and can be realized by means of a computer algebra program. Therefore the influence of numerical errors is greatly reduced.

As an example, we first excite the antenna by the two frequencies $f_{1}=70 \mathrm{MHz}$ and $f_{2}=80 \mathrm{MHz}$. After four Picard iterations the amplitudes of the major frequencies in the emerging spectrum of the current through the nonlinear load

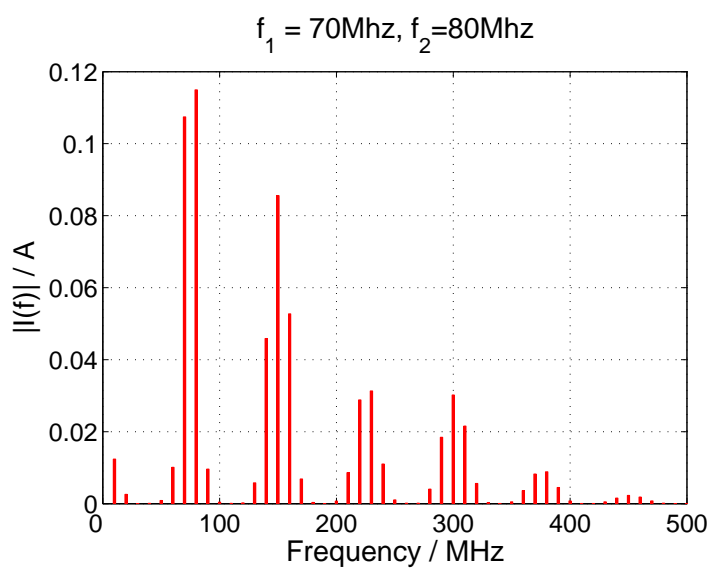

Fig. 7. Resulting spectrum of the current $I(\omega)$ through the nonlinear antenna load. The chosen two-tone excitation consists of the frequencies $f_{1}=70 \mathrm{MHz}$ and $f_{2}=80 \mathrm{~Hz}$. Significant contributions to the current at intermodulation frequencies are clearly visible.

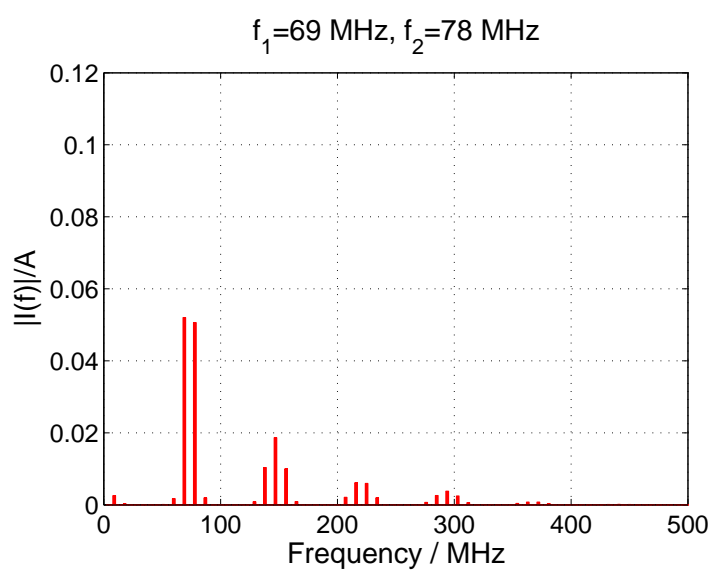

Fig. 8. Resulting spectrum of the current $I(\omega)$ through the nonlinear antenna load if the two-tone excitation consists of the (resonant) frequencies $f_{1}=69 \mathrm{MHz}$ and $f_{2}=78 \mathrm{MHz}$. If compared to the spectrum of Fig. 7 the amplitudes are considerably reduced.

do no longer significantly change and the corresponding result is shown in Fig. 7.

The chosen frequencies $f_{1}=70 \mathrm{MHz}$ and $f_{2}=80 \mathrm{MHz}$ are slightly above the resonance frequencies $f_{130}$ and $f_{310}$, respectively. To change this situation we lower the exciting frequencies and choose $f_{1}=69 \mathrm{MHz}$ and $f_{2}=78 \mathrm{MHz}$ such that these resonance frequencies are approached. Figure 8 shows the current spectrum which results after four Picard iterations. It is seen that the current amplitudes are considerably reduced. An explanation of this phenomenon is that the cavity resonances, which are represented by the parallel $R L C$-circuits of Fig. 6, act like filters that suppress the exciting frequencies. A more detailed discussion of this result can be found in (Gronwald, 2006). 
Nonlinear canonical problems, like the example just considered, clearly are important in the context of Electromagnetic Compatibility since they allow to calculate electric current and electromagnetic field spectra within systems that are subject to EMI fields. The corresponding techniques, as outlined above, are not trivial, though, and require in many respects a careful analyis.

\section{Conclusions}

In areas such as EMC analysis, network representations of electromagnetic field problems often are useful or even necessary. For systems with an infinite number of natural frequencies these representations are not unique. They must be chosen such that the occuring series expressions have good convergence properties. In field theory, this difficulty relates to the problem of choosing a quickly convergent electromagnetic Green's function. If antennas in resonating systems are considered the Green's function must efficiently represent both the Coulomb interaction and the resonances. In this respect it is often useful to split off the free space Green's function from the modal representation of the corresponding Green's function. As a result, a suitable network representation will contain network elements which either characterize free space properties or characterize resonances. It finally has been exemplified that the network representation of an antenna within a cavity can be used to calculate current spectra that originate from the intermodulation of primary frequencies at a nonlinear load.

\section{References}

Baum, C. E.: Single Port Equivalent Circuits for Antennas and Scatterers, Interaction Note 295, 1976.

Baum, C. E.: General Properties of Antennas, IEEE Trans. Electromagn. Compat., 44, 180-24, 2002.

Collin, R. E.: Field Theory of Guided Waves, 2nd ed., IEEE Press, New York, 1997.

Elliott, R. S.: Antenna theory and design, Prentice-Hall, Englewood Cliffs, 1981.
Felsen, L. P. (ed.): Hybrid Formulation of Wave Propagation and Scattering, Martinus Nijhoff, Dordrecht, 1984.

Foster, R. M.: A reactance theorem, Bell Sys. Tech. Jour., 3, 259267, 1924.

Gronwald, F.: Calculation of mutual antenna coupling within rectangular enclosures, IEEE Trans. Electromagn. Compat., 47, 1021-1025, 2005.

Gronwald, F: Antenna Theory in Resonating Systems derived from Fundamental Electromagnetism, Habilitation Thesis, (Ottovon-Guericke-University Magdeburg), diglib.uni-magdeburg.de/ Dissertationen/2006/fragronwald.htm, 2006.

Haase, H., Nitsch, J., and Steinmetz, T.: Transmission-Line Super Theory: A New Approach to an Effective Calculation of Electromagnetic Interactions, URSI Radio Science Bulletin (Review of Radio Science), 307, 33-60, 2003.

Haase, H., Steinmetz, T., and Nitsch, J.: New propagation models for electromagnetic waves along uniform and nonuniform cables, IEEE Trans. on Electromagn. Compat., 46, 345-352, 2004.

Huang, Y., Narayanan, R. N., and Kadambi, G. R.: Equivalent Circuit for Antenna-Cavity Coupling in Wheeler's Cap Technique, Electromagnetics, 25, 115-131, 2005.

Lee, K. S. H. (ed.): EMP Interaction: Principles, Techniques, and Reference Data, revised printing, Taylor \& Francis, Washington D.C., 1995.

Maas, S. A.: Nonlinear Microwave Circuits, 2nd ed., Artech House, Norwood, 2003.

Morse, P. M. and Feshbach, H.: Methods of Theoretical Physics I+II, McGraw Hill, New York, 1953.

Nitsch, J., Korovkin, N., Solovieva, E., and Gronwald, F.: Characterization and Compensation of Beat-Induced Intermodulation Effects in Analog Circuits, ANALOG '05, 8. GMM/ITGDiskussionssitzung, Hannover, 191-196, 2005.

Ramo, S., Whinnery, J. R., and Van Duzer, T.: Fields and Waves in Communication Electronics, John Wiley \& Sons, New York, 1994.

Schelkunoff, S. A.: Representation of Impedance Functions in Terms of Resonant Frequencies, Proc. of the I.R.E., 32, 83-90, 1944.

Tesche, F. M., Ianoz, M. V., and Karlsson, T.: EMC Analysis Methods and Computational Methods, John Wiley \& Sons, New York, 1997. 